\title{
Acabados para madera en exteriores
}

\author{
Ma. Iracema Castillo Morales ${ }^{1}$ \\ José Javier Erdoiza Sordo
}

\section{RESUMEN}

La madera que se encuentra sometida al intemperismo (lluvia, viento, luz solar, etc), llega a sufrir alteraciones a nivel superficial presentado una apariencia grisácea cuando el ataque es muy severo, facilitando el camino para que otro agente que la deteriora internamente actúe. Para evitar este tipo de problemas, es necesario protegerla con una mezcla de substancias químicas llamadas acabados 0 recubrimientos superficiales. Se probaron 5 tipos d acabados; de los cuales dos forman película (barnices y pinturas) y los tres restantes penetran en la madera (repelentes al agua, tintas y preservadores hidrosolubles). Se comprobó que las tintas fueron las más efectivas para proteger a la madera de los diferentes cambios de clima.

\section{PALABRAS CLAVE}

Intemperismo, acabados, repelentes al agua, tintas, barnices, pinturas, sales hidrosolubles.

\begin{abstract}
Wood exposed to weathering (rain, wind, sunlight, etc.) can suffer surface damage, which is characterized by a greyish appearance when the exposure is severe. In turn, this can lead to invasion by other agents that can easily cause internal deterioration of the wood. To avoid this problem, it is necessary to protect the wood by applying a mixture of chemical products called finishes or surface coatings. Five kinds of finishes were tested, two of which (varnishes and paints) form a film, while the other three (water repellents, stains, and waterborne preservatives) penetrate the wood. It was demonstrated that stains are more effective at protecting wood from climatic exposure tan the other treatments.
\end{abstract}

\section{KEY WORDS}

Weathering, wood finishes, water repellents, stains, varnishes, paints, waterborne salts.

1 Técnico Académico. Departamento de Productos Forestales y Conservación de Bosques. Instituto de Ecología, A.C. Apdo. Postal 63. Xalapa, Ver., 91000 México.

2 SEQUOIA (Asesoría y Tratamiento de Maderas),8 poniente Núm.118 Col. Ferrocerrilera. Xalapa, Ver., México.

Manuscrito recibido para su publicación el 18 de Enero de 1994. 


\section{INTRODUCCION}

El intemperismo es uno de los agentes físicos que deterioran la madera, y es causado por las condiciones climáticas del medio ambiente como lluvia, viento y luz solar. Es un fenómeno que ataca solo a las capas superficiales (aproximadamente se descasta $0.1 \mathrm{~mm}$ del grosor de la madera por año), cambiando la apariencia natural de la madera. Por otro lado, puede ser una de las causas para que otro tipo de agentes biológicos (microorganismos, hongos, bacterias) la dañen profundamente. Los factores que provocan este fenómeno son: las radiaciones ultravioleta, el calor, humedad y la contaminación atmosférica (USFPL, 1966).

De todos los factores que ocasionan intemperismo, los fotones emitidos por las radiaciones solares, son los que más dañan a la madera, provocando una amplia gama de variaciones químicas en su superficie. Estas alteraciones son producidas sobre los polímeros naturales que la constituyen y cuya susceptibilidad varía debido a la presencia de grupos funcionales llamados cromofóricos (carbonilos, carboxilos, quinonas, dobles ligaduras conjugadas).

La energía absorbida, provoca una disolución de las uniones que forman a las moléculas de celulosa, hemicelulosas, lignina y algunos extractivos. El proceso homolítico forma radicales libres como productos primarios fotoquímicos. Este factor, independiente de la participación del oxígeno y del agua puede dar origen a una despolimerización que lleva a la decoloración y degradación superficial de la madera (IPT, 1986).
La primera etapa del proceso es la decoloración, amarillamiento u oscurecimiento de la madera, debido a la solubilidad de los extractivos presentes en las células de algunas especies, seguida de la separación de las fibras, ocasionada principalmente por las radiaciones ultravioleta (U.V.) del sol, que descomponen a la lignina (componente cementante), provocando la lixiviación de éstas por la acción de la lluvia (Cassens y Feist, 1986).

Por otra parte, el intemperismo envuelve también un mecanismo de fotooxidación de los grupos hidroxilos $(\mathrm{OH})$ de la celulosa y hemicelulosas, ya que se han obtenido cantidades significativas de bióxido de carbono $\left(\mathrm{CO}_{2}\right)$, monóxido de carbono (CO) y metanol como productos de degradación, cuando la madera está expuesta a la luz U.V. y al viento (USFPL, 1966).

Además, la humedad produce cambios en la anatomía de la superficie de las células e induce a la formación de grietas causadas por la contracción e hinchamiento periódico o cíclico de la madera, mientras que el calor acelera la velocidad de las reacciones químicas que se llevan a cabo (Cassens y Feist, 1986). Otro factor es la contaminación atmosférica que en los últimos años ha adquirido importancia en donde se da, ya que involucra el (anhídrido de nitrógeno IV $\left(\mathrm{NO}_{2}\right)$, anhídrido de azufre IV $\left(\mathrm{SO}_{2}\right)$ y el ozono $\left(\mathrm{O}_{3}\right)$, provocando el ablandamiento de la lignina.

En la tabla 1 se muestra la degradación ocasionada a la madera por los diferentes tipos de energía cuando está sometida a las condiciones del intemperismo (Schniewind,1989). 
Tabla 1. Degradación de la madera ocasionada por diferentes tipos de energías

\begin{tabular}{|c|c|c|}
\hline ENERGIA & DEGRADACION & NIVEL \\
\hline Calor & Oscurecimiento & Ligero \\
\hline \multirow{3}{*}{ Radiaciones U.V. } & Cambio de color & Severo \\
\cline { 2 - 3 } & Degradación química & Severo \\
\hline \multirow{4}{*}{ Mecánica } & Desgaste & Ligero \\
\cline { 2 - 3 } & Erosión & Ligero \\
\cline { 2 - 3 } & Agrietamiento & Severo \\
\cline { 2 - 3 } & Separación de fibras & Severo \\
\hline \multirow{5}{*}{ Química } & Agrietamiento & Severo \\
\cline { 2 - 3 } & Separación de fibras & Severo \\
\cline { 2 - 3 } & Lixiviación & Severo \\
\cline { 2 - 3 } & Cambio de color & Severo \\
\cline { 2 - 3 } & Pérdida de resistencia & Severo \\
\hline
\end{tabular}

\section{PROTECCION CONTRA EL INTEMPERISMO}

Para disminuir todos estos problemas es necesario proteger a la madera con sustancias adecuadas llamadas: acabados, los cuales se clasifican en dos tipos:

1) Los que penetran a través de la superficie de la madera.

2) Los que forman una película recubriendo la superficie de la madera.

\section{Los que penetran}

Se clasifican en transparentes (repelentes al agua) y pigmentados (tintas y preservadores hidrosolubles).
Repelentes al agua

Como su nombre lo indica, son soluciones que proporcionan a la madera la capacidad de repeler al agua, protegiéndola contra agentes degradadores (hongos, insectos, intemperismo) y que reducen el agrietamiento y sólo existe un pequeño cambio (oscurecimiento) en el color original; pero que mantiene el grano visible.

Están formados por un funguicida (pentaclorofenol, naftanato de cobre y naftanato de zinc), un solvente (thinner) y los ingredientes que evitan la salida del fungicida y repelen el agua (aceite de linaza y cera). 
Tintas

Son soluciones que disminuyen la degradación ocasionada por la radiación de la luz ultravioleta, cambiando el color y acentuando el grano o veteado de la madera. Una de las ventajas de las tintas es que como no forman película, no existen problemas de burbujas y descarapelamiento del acabado; por lo que facilita el mantenimiento después de un tiempo de aplicadas a la madera.

Este tipo de acabado está formado, además de los mismos ingredientes de los repelentes al agua, por estabilizadores orgánicos o inorgánicos llamados pigmentos y aditivos, que se le agregan para mejorar la dispersión y estabilidad de los mismos por ejemplo es estearato de zinc.

\section{Preservadores hidrosolubles}

Son soluciones preservadoras que han dado buenos resultados como acabados. En su composición intervienen sustancias fungicidas y fijadoras (sales de cobre, arsénico y cromo), además del agua que se utiliza como solvente. La madera adquiere una coloración verde, quedando protegida contra agentes que la deterioran como los hongos, insectos y el intemperismo (Feist, et al., 1977).

\section{Los que forman película}

Estos acabados forman una película superficial en la madera y pueden ser de dos tipos: barnices y pinturas.

\section{Barnices}

Productos compuestos por aglutinantes, plastificantes, disolventes y diluyentes que al ser aplicados originan una película insoluble en agua, adherente y dura, generalmente lisa y brillante. Los barnices son transparentes y permiten conservar la apariencia natural de la madera.
El aglutinante: es la parte principal del barniz que se queda sobre el objeto, después de secarse o endurecerse la película. Ejemplos: nitrocelulosa y poliester.

El plastificainte: confiere flexibilidad permanente a las capas formadas por el barniz. Ejemplos: ftalato de butilo, tricresilfosfato y dioctilftalato.

El disolvente: líquido simple o mixto, volátil que se emplea para dispersar el aglutinante. Ejemplos: acetato de etilo, actato de metilo, metil-etil cetona y ciclohexanona.

El diluyente: líquido simple o mixto, volátil, miscible en el medio de suspensión, mejorando con esto las características de aplicación (no disuelve el aglutinante). Ejemplos: alcohol isopropílico, alcohol butílico y tolueno.

\section{Pinturas}

Una pintura es una formulación constituída por una materia sólida que imparte color, suspendida en un medio líquido y aplicada como recubrimiento a diversos tipos de superficies. Las pinturas están constituidas por los mismos ingredientes que los barnices, agregándoles un pigmento y materias de carga (sustancias que ayudan a la incorporación del pigmento) (Vélez, 1965).

\section{OBJETIVO}

Determinar la efectividad de 16 acabados (12 que penetran y 4 que sólo forman una película superficial), utilizados para proteger a la madera sometida a diferentes condiciones climáticas (intemperismo). 


\section{METODOLOGIA}

\section{Características de las probetas}

Las medidas de las tablas utilizadas fueron de $2.5 \times 16 \times 61 \mathrm{~cm}$ con un contenido de humedad del $18 \%$, aproximadamente, utilizándose madera de pino. Se marcaron con números y letras para diferenciar el tipo de acabado, el número de probeta y si se encontraba o no cepillada la madera (Fig. 1). Al cortarlas, se obtuvieron 7 probetas cepilladas y 7 sin cepillar por acabado incluyendo el control, dando un total de 238 muestras.

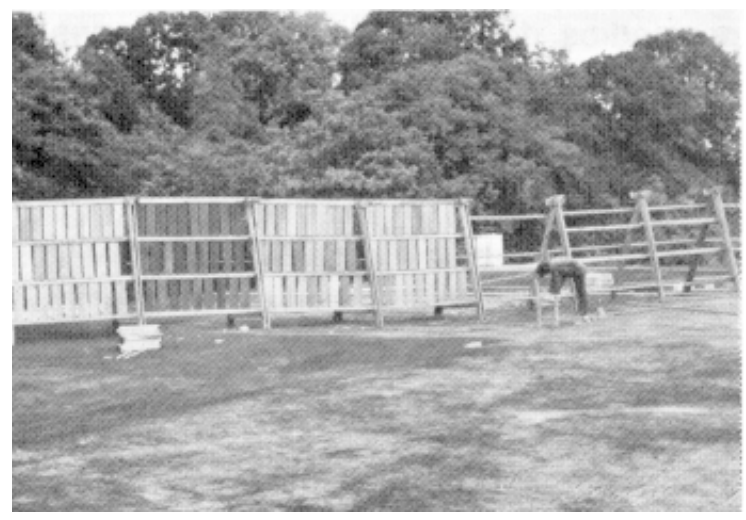

Figura 1. Aspecto general de los especímenes al inicio de la prueba

\section{Acabados}

A continuación se describen los acabados utilizados:

\section{a) Los que penetran}

- Repelentes al agua

1R (cera, thinner, pentaclorofenol, aceite de linaza); $\mathbf{2 R}$ (cera, thinner, pentraclorofenol, naftanato de zinc, naftanato de cobalto); 12J (cera, thinner, pentaclorofenol, japan).

\section{- Tintas}

3T (cera, thinner, pentaclorofenol, aceite de linaza, estearato de zinc, rojo francés); 4T (cera, thinner, pentaclorofenol, aceite de linaza, estearato de zinc, tierra casel); 5T (cera, thinner, pentaclorofenol, aceite de linaza, ocrillo francés); 6T (cera, thinner, pentaclorofenol, barniz de uretano, óxido de cromo); 13J (cera, thinner, pentaclorofenol, japan, estearato de zinc, tierra casel).

\section{- Preservadores hidrosolubles}

7S (hidróxido de amonio, agua, sulfato de cobre II pentahidratado, dicromato de potasio dihidratado, ácido cítrico); $\mathbf{8 S}$ (hidróxido de amonio, agua, pentaclorofenato de sodio); 95 (hidróxido de amonio, agua, sulfato de cobre II pentahidratado, dicromato de potasio dihidratado, ácido arsénico); 10S (hidróxido de amonio, agua, sulfato de cobre II pentahidratado, cromato de sodio tetrahidratado).

b) Los que forman película

- Barnices

14B (Barniz marino)

- Pinturas

15P (aceite): 16P (epóxica); 17P (poliuretano)

\section{Aplicación de los acabados}

Los repelentes al agua, tintas y sales inorgánicas se aplicaron por inmersión; mientras que para el barniz y las pinturas, se utilizó brocha, aplicándoles 2 manos o capas, en todas las superficies, dejando el tiempo necesario para su secado entre cada aplicación.

\section{Tratamiento final}

Se construyeron estructuras de madera tratada con sales de ACC (ácidocobre-cromo), en donde se colocaron las muestras ubicadas en lugares sin protección (al aire libre), para favorecer así 
las condiciones de intemperismo (en la azotea del edificio del Departamento de Productos Forestales) (Fig. 2). Las muestras se revisaron periódicamente cada 4 meses durante 17 meses.

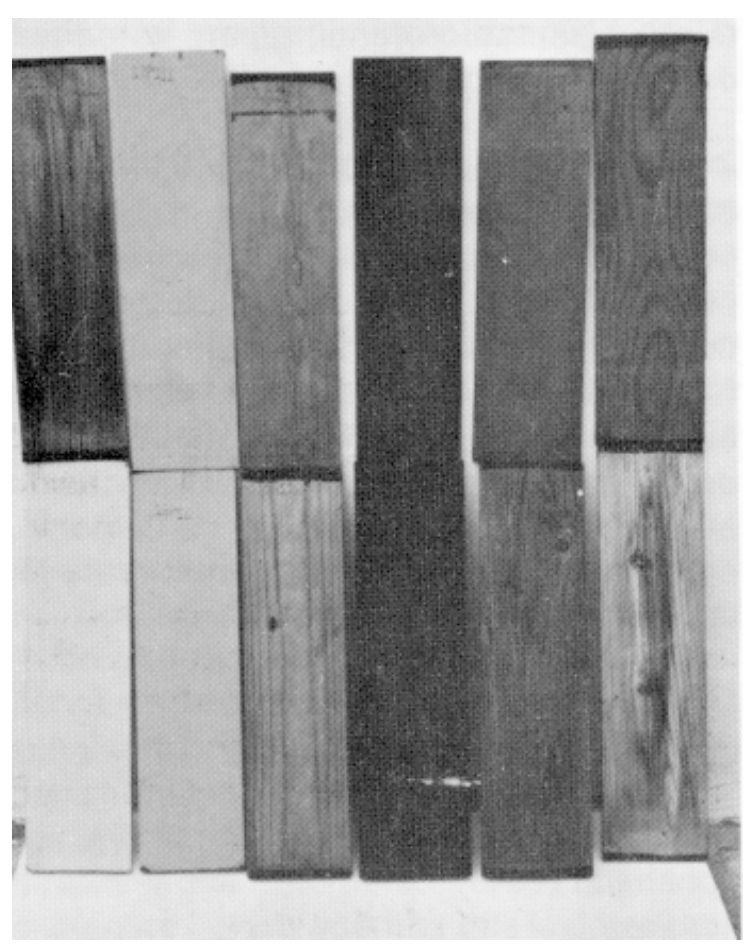

Figura 2. Especímenes después de 17 meses de exposición

\section{Calificación de las muestras}

Las probetas se revisaron individualmente, conforme a las normas de American Society for Testing Materials (ASTM 1986a, b, c y d; 1987a y b; y 1988), y las escalas elaboradas en el Departamento indicadas en las tablas 2 y 3. De las primeras, se calificaron manchado, erosión, presencia de escamas, burbujas, fisuras y grietas. Las escalas del Departamento se utilizaron para alabeo, cambios de color, apariencia general, nudos y resina. Las observaciones se realizaron tanto al sustrato madera como a la superficie del acabado, agrupándose de la siguiente manera:

\section{SUSTRATO SUPERFICIE DEL ACABADO}

$\begin{array}{ll}\text { Grietas } & \text { Decoloración } \\ \text { Alabeo } & \text { Manchado } \\ \text { Nudos } & \text { Grietas } \\ \text { Resina } & \text { Escamas y polvo } \\ & \text { Apariencia general }\end{array}$

\section{RESULTADOS}

Las tablas 4 y 5 indican los resultados obtenidos de las muestras sin cepillar y cepilladas, utilizándose solamente los promedios de todas las calificaciones y comparándolas con la escala que se menciona en la parte inferior de cada tabla.

En la tabla 6 se muestran los resultados obtenidos con la madera sin cepillar agrupando los acabados aplicados, observándose que para el repelente al agua $2 R$, en el que no se usó aceite de linaza empezó a fallar al séptimo mes mientras que para los otros dos repelentes (1R, 12J), la presencia del intemperismo fue al décimo mes de exposición.

En el caso de las tintas, tres de ellas (5T, 6T y 13J), fallaron al séptimo mes, mientras que $3 \mathrm{~T}$ y $4 \mathrm{~T}$, las cuales contaban con todos los ingredientes necesarios, como el estearato de zinc que ayuda a la mejor incorporación del pigmento, al décimo séptimo mes todavía se mantenía en buenas condiciones.

Para el caso de las sales inorgánicas (7S, 8S, 9S y 10S), todas presentan características de intemperismo desde el cuarto mes, debido a que la concentración de la solución utilizada fue baja (2\%). 
Tabla 2. Normas de American Society for Testing and Materials que se utilizaron para calificar los acabados para madera

\begin{tabular}{|l|l|}
\hline \multicolumn{1}{|c|}{ CLAVE } & \multicolumn{1}{c|}{ NORMA } \\
\hline ASTM D3274-82 (ASTM, 1988) & $\begin{array}{l}\text { Evaluating degree of surface disfigurement of paint } \\
\text { films by microbial (fungal or algal) growth or soil and } \\
\text { dirt accumulation. }\end{array}$ \\
\hline ASTM D 772-86 (ASTM, 1986a) & Evaluating degree of flaking (scaling) of exterior paints \\
\hline ASTM D 659-86 (ASTM, 1986b) & Evaluating degree of chalking of exterior paints. \\
\hline ASTM D 714-87 (ASTM, 1987a) & Evaluating degree of blistering of paints. \\
\hline ASTM D 660-87 (ASTM, 1987b) & Evaluating degree of checking of exterior paints. \\
\hline ASTM D 661-86 (ASTM, 1986c) & Evaluating degree of cracking of exterior paints. \\
\hline ASTM D 662-86 (ASTM, 1986d) & Evaluating degree of erosion of exterior paints. \\
\hline
\end{tabular}

Para los grupos que forman película como en el caso del barniz marino, que era el disponible para exteriores, empezó a fallar desde el cuarto mes, presentando descarapelamiento de las capas aplicadas.

Las pinturas que se utilizaron fueron de aceite, epóxica y de poliuretano, la primera no presentó características severas de intemperismo hasta el final de la prueba (17 meses), en cambio la epóxica empezó a fallar al séptimo mes. Finalmente en el caso de la pintura de poliuretano no se concluyó la prueba debido a la desaparición de las muestras, observándose sólo hasta el séptimo mes pocos cambios en el acabado.

Por otro lado, tanto para el caso de los barnices como de las pinturas, al ser cortados los especímenes, la mayoría presentó ataque por terminas. Todas las pruebas fueron comparadas con los especímenes que se tenían de testigo (11C), a las cuales no se les aplicó ningun tipo de protección y empezaron a fallar desde el segundo mes de exposición. En la tabla 7 , se muestran las pruebas con la madera cepillada, observándose los mismos resultados que para la madera que no había sido cepillada (tabla 6).

\section{CONCLUSIONES}

Los acabados que más duraron fueron las tintas, sobre todo aquellas a las que se les agregó como ingrediente la sustancia capaz de incorporar el pigmento al resto de la solución, en este caso fue el estearato de zinc.

Las pinturas de aceite, poliuretano y epóxica se recomiendan en donde la apariencia natural de la madera no es tan importante, teniendo en consideración que no contienen fungicidas 0 insecticidas que protejan a ésta contra el ataque por degradadores tales como los hongos 0 insectos. 
Tabla 3. Escalas elaboradas en el Departamento de Productos Forestales y Conservación de Bosques para calificar acabados de maderas para exteriores

\begin{tabular}{|c|c|c|}
\hline DEFECTO & CALIFICACION & CARACTERISTICAS \\
\hline \multirow{6}{*}{$\begin{array}{l}\text { ALABEO } \\
\text { Se mide la } \\
\text { deformación }\end{array}$} & 10 & $0.0-0.99 \mathrm{~mm}$ \\
\hline & 8 & $1.00-3.99 \mathrm{~mm}$ \\
\hline & 6 & $4.00-7.99 \mathrm{~mm}$ \\
\hline & 4 & $8.00-11.99 \mathrm{~mm}$ \\
\hline & 2 & $12.00-15.99 \mathrm{~mm}$ \\
\hline & 0 & $16.00-+\mathrm{mm}$ \\
\hline \multirow{6}{*}{ NUDOS } & 10 & Sin nudos \\
\hline & 8 & Fijo en el área central \\
\hline & 6 & Fino y en el área media de la tabla \\
\hline & 4 & $\begin{array}{l}\text { Fijo que atraviese a la tabla en extremos u } \\
\text { orillas }\end{array}$ \\
\hline & 2 & Nudo flojo (no caido) \\
\hline & 0 & Nudo caido \\
\hline \multirow{3}{*}{ RESINA } & 10 & No presenta \\
\hline & 6 & Presenta y no exudó \\
\hline & 0 & Exudó \\
\hline \multirow{4}{*}{$\begin{array}{l}\text { APARIENCIA } \\
\text { GENERAL }\end{array}$} & 10 & No cambió \\
\hline & 8 & Cambio ligero del sustrato o acabado \\
\hline & 6 & $\begin{array}{l}\text { Condición en la cual el acabado deberá } \\
\text { aplicarse con una mínima preparación de la } \\
\text { superficie }\end{array}$ \\
\hline & 3 & $\begin{array}{l}\text { El acabado debe volverse a aplicar con una } \\
\text { preparación sustancial de la superficie } \\
\text { (presenta intemperismo) }\end{array}$ \\
\hline \multirow{4}{*}{$\begin{array}{l}\text { CAMBIO DE } \\
\text { COLOR } \\
\text { (tablas Munsell) }\end{array}$} & 10 & No existe cambio \\
\hline & 8 & 1 brinco \\
\hline & 4 & 2 brincos \\
\hline & 2 & 3 brincos o más \\
\hline
\end{tabular}


Tabla 4. Acabados para madera en exteriores (madera sin cepillar)

\begin{tabular}{|c|c|c|c|c|c|c|c|c|c|c|c|c|}
\hline GRUPO & \multicolumn{2}{|c|}{2 MES } & \multicolumn{2}{|c|}{4 MES } & \multicolumn{2}{|c|}{7 MES } & \multicolumn{2}{|c|}{10 MES } & \multicolumn{2}{|c|}{13 MES } & \multicolumn{2}{|c|}{17 MES } \\
\hline 1R & 9.7 & $E$ & 9.7 & $E$ & 6.6 & $B$ & 6.2 & $\mathrm{M}$ & 5.6 & $M$ & 5.2 & $M$ \\
\hline $2 R$ & 9.7 & $E$ & 9.7 & $E$ & 5.6 & $\mathrm{M}$ & 4.1 & $\mathrm{M}$ & 5.3 & $M$ & 5.3 & $M$ \\
\hline $12 \mathrm{~J}$ & 9.7 & $E$ & 9.5 & $E$ & 8.7 & $\mathrm{MB}$ & 7.6 & $B$ & 6.1 & $M$ & 5.6 & $M$ \\
\hline 3T & 9.7 & $E$ & 9.6 & $E$ & 8.3 & $\mathrm{MB}$ & 8.3 & $\mathrm{MB}$ & 8.3 & $\mathrm{MB}$ & 7.5 & $B$ \\
\hline 4T & 9.9 & $E$ & 9.6 & $E$ & 9.6 & $E$ & 9.3 & $\mathrm{MB}$ & 8.5 & $\mathrm{MB}$ & 8.3 & $\mathrm{MB}$ \\
\hline $5 \mathrm{~T}$ & 9.3 & $\mathrm{MB}$ & 6.5 & $B$ & 6.1 & $\mathrm{M}$ & 5.3 & $\mathrm{M}$ & 5.2 & $\mathrm{M}$ & 5.2 & $\mathrm{M}$ \\
\hline $6 \mathrm{~T}$ & 6.5 & B & 6.3 & $M$ & 5.9 & $M$ & 5.2 & $M$ & 4.9 & $M$ & 4.7 & $M$ \\
\hline 13J & 9.6 & $E$ & 9.6 & $E$ & 6.3 & $M$ & 5.3 & $M$ & 4.9 & $M$ & 5.1 & $M$ \\
\hline $7 S$ & 9.5 & $\mathrm{MB}$ & 6.2 & $M$ & 6.1 & $\mathrm{M}$ & 4.7 & $\mathrm{M}$ & 4.5 & $M$ & 4.6 & $M$ \\
\hline $8 S$ & 8.8 & $\mathrm{MB}$ & 7.4 & B & 5.9 & $M$ & --- & -- & --- & -- & --- & -- \\
\hline 9S & 8.8 & $\mathrm{MB}$ & 5.8 & $\mathrm{M}$ & 5.4 & $M$ & 4.5 & $M$ & 3.8 & $M$ & 4.3 & $M$ \\
\hline $10 S$ & 6.8 & $B$ & 6.1 & $M$ & 6.1 & $\mathrm{M}$ & 4.8 & $\mathrm{M}$ & 5.1 & $M$ & 4.6 & $M$ \\
\hline $11 C$ & 6.3 & $M$ & 5.4 & $\mathrm{M}$ & 4.4 & $\mathrm{M}$ & 3.3 & $\mathrm{M}$ & 4.2 & $M$ & 4.8 & $M$ \\
\hline 14B & 9.6 & $E$ & 6.4 & $M$ & 4.7 & $M$ & 4.3 & $\mathrm{M}$ & 4.4 & $M$ & 4.9 & $M$ \\
\hline 15P & 9.6 & $E$ & 9.2 & $\mathrm{MB}$ & 8.7 & $\mathrm{MB}$ & 8.4 & MB & 7.9 & $B$ & 9.0 & MB \\
\hline 16P & 9.8 & $E$ & 8.9 & $\mathrm{MB}$ & 6.1 & $M$ & 4.5 & $M$ & 3.8 & $M$ & 5.3 & $M$ \\
\hline 17P & 9.4 & $\mathrm{MB}$ & 9.0 & $\mathrm{MB}$ & 8.6 & $\mathrm{MB}$ & --- & -- & --- & -- & --- & -- \\
\hline
\end{tabular}

\section{ESCALA}

$10-9.5$ Excelente (E) no existe cambio

9.49-8.0 Muy bien (MB) cambio ligero

7.99-6.5 Bien (B) reaplicación con mínima preparación de la superficie

6.49-0.0 Mal (M) reaplicación con preparación sustancial de la superficie 
Tabla 5. Acabados para madera en exteriores (madera cepillada)

\begin{tabular}{|c|c|c|c|c|c|c|c|c|c|c|c|c|}
\hline GRUPO & \multicolumn{2}{|c|}{2 MES } & \multicolumn{2}{|c|}{4 MES } & \multicolumn{2}{|c|}{7 MES } & \multicolumn{2}{|c|}{10 MES } & \multicolumn{2}{|c|}{13 MES } & \multicolumn{2}{|c|}{17 MES } \\
\hline $1 R$ & 9.3 & MB & 8.6 & MB & 5.5 & M & 5.8 & M & 4.7 & $M$ & 4.8 & $M$ \\
\hline $2 R$ & 9.4 & MB & 9.4 & TVID & 5.9 & M & 5.3 & M & 4.8 & M & 4.7 & $M$ \\
\hline $12 \mathrm{~J}$ & 9.4 & $\mathrm{MB}$ & 9.2 & MB & 8.6 & MB & 7.5 & $B$ & 5.1 & M & 4.8 & $M$ \\
\hline 3T & 9.7 & $E$ & 9.7 & $E$ & 8.9 & MB & 8.2 & MB & 7.3 & B & 6.7 & B \\
\hline 4T & 9.5 & $\mathrm{E}$ & 9.7 & $E$ & 9.3 & MB & 8.9 & MB & 8.5 & MB & 7.7 & B \\
\hline $5 T$ & 7.3 & B & 6.3 & $M$ & 5.9 & M & 4.5 & M & 4.8 & M & 4.6 & M \\
\hline $6 T$ & 6.7 & B & 6.6 & B & 5.7 & M & 5.1 & M & 4.9 & M & 4.7 & $M$ \\
\hline $13 \mathrm{~J}$ & 9.5 & MB & 9.6 & MB & 6.1 & M & 4.7 & $M$ & 4.3 & $M$ & 4.8 & $M$ \\
\hline $7 S$ & 9.3 & MB & 6.4 & M & 6.0 & M & 5.1 & M & 4.5 & M & 4.7 & M \\
\hline $8 S$ & 9.1 & MB & 6.9 & B & 5.5 & M & --- & -- & --- & -- & --- & -- \\
\hline 9S & 9.3 & MB & 5.8 & $M$ & 5.4 & M & 4.8 & M & 4.1 & M & 4.3 & $M$ \\
\hline $10 S$ & 6.6 & B & 5.9 & M & 5.7 & M & 4.5 & M & 4.7 & M & 3.9 & $M$ \\
\hline $11 \mathrm{C}$ & 6.4 & $M$ & 5.6 & $M$ & 4.4 & $M$ & 4.4 & $M$ & 4.5 & M & 4.2 & $M$ \\
\hline 14B & 9.2 & MB & 6.3 & $M$ & 4.9 & M & 4.4 & $M$ & 4.1 & $M$ & 4.1 & $M$ \\
\hline 15P & 9.0 & MB & 9.4 & MB & 8.7 & MB & 8.5 & MB & 7.9 & B & 8.4 & MB \\
\hline $16 P$ & 9.7 & E & 8.3 & MB & 5.9 & M & 4.4 & M & 4.4 & M & 4.9 & M \\
\hline $17 \mathrm{P}$ & 8.9 & MB & 8.7 & MB & 8.4 & MB & --- & -- & --- & -- & --- & -- \\
\hline
\end{tabular}

ESCALA

$10-9.5 \quad$ Excelente $(\mathrm{E})$ no existe cambio

$9.49-8.0 \quad$ Muy bien (MB) cambio ligero

7.99 - $6.5 \quad$ Bien (B) reaplicación con una mínima preparación de la superficie

$6.49-0.0 \quad \mathrm{Mal}(\mathrm{M})$ reaplicación con preparación sustancial de la superficie 
Tabla 6. Acabados para madera en exteriores (madera sin cepillar)

\begin{tabular}{|c|c|c|c|c|c|c|}
\hline GRUPO & 2 MES & 4 MES & 7 MES & 10 MES & 13 MES & 17 MES \\
\hline $1 R$ & E & E & B & $M$ & $M$ & $M$ \\
\hline $2 R$ & $E$ & $E$ & $M$ & $M$ & $M$ & $M$ \\
\hline $12 \mathrm{~J}$ & $E$ & $E$ & MB & $B$ & $M$ & $M$ \\
\hline GRUPO & 2 MES & 4 MES & 7 MES & 10 MES & 13 MES & 17 MES \\
\hline 3T & $\mathrm{E}$ & $E$ & $\mathrm{MB}$ & $\mathrm{MB}$ & MB & B \\
\hline $4 T$ & $\mathrm{E}$ & E & $E$ & MB & MB & MB \\
\hline $5 T$ & MB & B & M & M & $M$ & M \\
\hline $6 T$ & B & $\mathrm{M}$ & $\mathrm{M}$ & $\mathrm{M}$ & $\mathrm{M}$ & $\mathrm{M}$ \\
\hline 13J & $E$ & $E$ & $M$ & $M$ & $M$ & $M$ \\
\hline GRUPO & 2 MES & 4 MES & 7 MES & 10 MES & 13 MES & 17 MES \\
\hline $7 S$ & MB & $M$ & $M$ & $M$ & M & $M$ \\
\hline $8 S$ & MB & B & $M$ & -- & -- & -- \\
\hline $9 S$ & MB & $M$ & $M$ & $M$ & $M$ & $M$ \\
\hline $10 S$ & B & $M$ & $M$ & $M$ & $M$ & M \\
\hline GRUPO & 2 MES & 4 MES & 7 MES & 10 MES & 13 MES & 17 MES \\
\hline $11 C$ & M & $\mathrm{M}$ & $M$ & $\mathrm{M}$ & $\mathrm{M}$ & $M$ \\
\hline GRUPO & 2 MES & 4 MES & 7 MES & 10 MES & 13 MES & 17 MES \\
\hline 14B & $E$ & $M$ & $M$ & $M$ & $M$ & $M$ \\
\hline GRUPO & 2 MES & 4 MES & 7 MES & 10 MES & 13 MES & 17 MES \\
\hline $15 P$ & $E$ & $\mathrm{MB}$ & $\mathrm{MB}$ & $\mathrm{MB}$ & $B$ & MB \\
\hline $16 \mathrm{P}$ & $E$ & $\mathrm{MB}$ & $M$ & $M$ & $M$ & $M$ \\
\hline $17 P$ & MB & $\mathrm{MB}$ & MB & -- & -- & -- \\
\hline
\end{tabular}


Tabla 7. Acabados para madera en exteriores (madera cepillada)

\begin{tabular}{|c|c|c|c|c|c|c|}
\hline GRUPO & 2 MES & 4 MES & 7 MES & 10 MES & 13 MES & 17 MES \\
\hline $1 R$ & MB & MB & M & $M$ & $M$ & $\mathrm{M}$ \\
\hline $2 R$ & MB & MB & M & $M$ & $\mathrm{M}$ & $\mathrm{M}$ \\
\hline $12 \mathrm{~J}$ & MB & MB & MB & B & M & M \\
\hline GRUPO & 2 MES & 4 MES & 7 MES & 10 MES & 13 MES & 17 MES \\
\hline $3 T$ & $E$ & $E$ & MB & MB & $M$ & M \\
\hline $4 T$ & $E$ & $E$ & MB & MB & MB & B \\
\hline $5 \mathrm{~T}$ & $B$ & $M$ & $M$ & $M$ & $M$ & M \\
\hline $6 \mathrm{~T}$ & B & B & M & M & M & M \\
\hline $13 \mathrm{~J}$ & MB & MB & M & $\mathrm{M}$ & M & M \\
\hline GRUPO & 2 MES & 4 MES & 7 MES & 10 MES & 13 MES & 17 MES \\
\hline $7 S$ & MB & $M$ & M & $\mathrm{M}$ & M & $\mathrm{M}$ \\
\hline $8 S$ & MB & $M$ & M & -- & -- & -- \\
\hline $9 S$ & MB & $M$ & M & $M$ & $M$ & M \\
\hline $10 S$ & B & M & M & $\mathrm{M}$ & $M$ & M \\
\hline GRUPO & 2 MES & 4 MES & 7 MES & 10 MES & 13 MES & 17 MES \\
\hline $11 \mathrm{C}$ & $M$ & $M$ & M & $M$ & $M$ & M \\
\hline GRUPO & 2 MES & 4 MES & 7 MES & 10 MES & 13 MES & 17 MES \\
\hline 14B & MB & M & M & $M$ & $M$ & M \\
\hline GRUPO & 2 MES & 4 MES & 7 MES & 10 MES & 13 MES & 17 MES \\
\hline $15 P$ & MB & MB & MB & MB & $B$ & MB \\
\hline $16 P$ & $E$ & MB & M & $\mathrm{M}$ & M & $\mathrm{M}$ \\
\hline 17P & MB & MB & MB & -- & -- & -- \\
\hline
\end{tabular}


El barniz marino utilizado en esta prueba, no es recomendable para la madera que va a estar expuesta a las condiciones de intemperismo, debido a que la mayoría de las muestras presentan descarapelamiento.

Finalmente, se recomiendan los acabados que no forman película, como los repelentes al agua y las tintas, con su respectivo mantenimiento.

\section{RECONOCIMIENTOS}

La elaboración de este manuscrito se realizó en el Departamento de Productos Forestales y Conservación de Bosques del Instituto de Ecología, A. C. con recursos fiscales con la clave 902-13.

\section{REFERENCIAS}

American Society for Testing and Materials. 1986a. Annual Book of ASTM Standards. Sección 6 Vol. 06.01 Paints, related coatings, and aromatics D772-86. Evaluating degree of flaking (scaling) of exterior paints. Phyladelphia, Pa. pp: 97-100.

American Society for Testing and Materials. 1986b. Annual Book of ASTM Standards. Sección 6 Vol. 06.01 Paints, related coatings, and aromatics D659-86. Evaluating degree of chalking of exterior paints. Philadelphia, Pa. pp: 70-71.

American Society for Testing and Materials. 1986c. Annual Book of ASTM Standards. Sección 6 Vol. 06.01 Paints, related coatings, and aromatics D661-86. Evaluating degree of cracking of exterior paints. Philadelphia, Pa. pp: 82-83. 06.01 Paints, related coatings, and aromatics D661-86. Evaluating degree of cracking of exterior paints. Philadelphia, Pa. pp: 82-83.

American Society for Testing and Materials. 1986d. Annual Book of ASTM Standards. Sección 6 Vol. 06.01 Paints, related coatings, and aromatics D662-86. Evaluating degree of erosion of exterior paints. Philadelphia, Pa. pp: 84-85.

American Society for Testing and Materials. 1987a. Annual Book of ASTM Standards. Sección 6 Vol. 06.01 Paints, related coatings, and Aromatics D714-87. Evaluating degree of blistering of paints. Philadelphia, Pa. pp: 91-96.

American Society for Testing and Materials. 1987b. Annual Book of ASTM Standards. Sección 6 Vol. 06.01 Paints, related coatings, and Aromatics D660-87. Evaluating degree of checking of exterior paints. Philadelphia, Pa. pp: 73-81.

American Society for Testing and Materials. 1988. Annual Book of ASTM Standards. Sección 6 Vol. 06.01 Paints, related coatings, and Aromatics D3274-82. Evaluating degree of surface disfigurement of paint films by microbial (fungal or algal) growth or soil accumulation. Philadelphia, Pa. pp: 473-475.

Cassens, D. y W. Feist. 1986. Finishing wood exteriors. Selection, application and maintenance. USDA, Forest Service, Agriculture Handbook No. 647. Madison, Wis. 56 pp.

Feist, W., E. Mraz y J. Black. 1977. Durability of exterior wood stains. Forest Products Journal 27(1): 13-16. 
Instituto de Pesquisas Tecnológicas (IPT). 1986. Manual de preservacâo de madeiras. V.1. Secretaría de Industria, Comercio, Ciência e Tecnología. Sâo Paulo, Brasil. pp: 193-207.

Schniewind, A.P. 1989. Concise encyclopedia of wood \& wood-based materials. Pergamon Press. Cambridge, Mass. pp: 325-326.
US Forest Products Laboratory. 1966. Wood finishing: weathering of wood. USDA, Forest Service, Forest Products Lab., FPL-0135. Madison, Wis. 4 pp.

Vélez, M.R. 1965. Pinturas y barnices para la madera. CTBA, AITIM. Cuaderno No. 61. Madrid, España. $63 \mathrm{pp}$. 